This article has appeared in Nonlinearity 11 (1998) 529-545

\title{
All possible chaotic dynamics can be approximated in three dimensions
}

\author{
James C. Robinson* \\ Department of Applied Mathematics and Theoretical Physics, \\ University of Cambridge, \\ Silver Street, Cambridge, CB3 9EW, U.K.
}

\begin{abstract}
It is known that neither one- nor two-dimensional ordinary differential equations can exhibit chaotic phenomena, while such behaviour is possible in threedimensional systems. Here it is shown that the solutions of any dissipative equation can be approximated arbitrarily closely by the trajectories of an appropriate threedimensional system. This class includes the Navier-Stokes equations, showing that fully developed turbulent flows can be approximated using just three degrees of freedom.
\end{abstract}

AMS classification scheme numbers: 34C35, 34D40, 34D45, 35B35, 35B40, 76F20

\footnotetext{
* email: j.c.robinson@damtp.cam.ac.uk
} 


\section{Introduction}

Investigation of the complex behaviour arising from coupled sets of ordinary differential equations has been greatly advanced in the last three decades by the increasing use of computer-generated pictures. These provide some intuition about such systems, and have led to many new results.

However, due to the classical Poincaré-Bendixson theorem (see, for example, Katok and Hasselblatt 1995), any recurrent trajectory in a planar two-dimensional flow must be periodic. So three-dimensional systems are certainly necessary to obtain more complicated behaviour. That they are sufficient is shown by various model equations (see, for example, Glendinning 1988).

It is shown in this paper that the dynamics of any dissipative system (one whose orbits are eventually bounded) can be recovered, to within an arbitrarily small error, from the solutions of an appropriate three-dimensional set of equations. The result can also be applied to the asymptotic behaviour of many partial differential equations, which are a priori infinite-dimensional systems, including the Navier-Stokes equations which govern fluid flow. In this way, turbulence can be approximated by a system with only three degrees of freedom.

The important conclusion is that numerical investigation of smooth threedimensional systems, which can be easily visualised, should provide some intuition about the asymptotic behaviour of systems in any dimension.

The paper is organised as follows. The first section introduces the problem in the context of ordinary differential equations, and the second section states the main theorem along with a sketch of the proof. Section 3 contains two results which will be important in the proof proper, which is given in section 4 . Section 5 covers 
the application to partial differential equations, treating an abstract parabolic equation as an example, and section 6 treats both the two- and three-dimensional Navier-Stokes equations in detail. Finally, section 7 considers some of the open problems that the result raises.

\section{Dissipative ordinary differential equations}

Consider the ordinary differential equation in $\mathbb{R}^{n}$,

$$
d u / d t=F(u) \quad u \in \mathbb{R}^{n} \quad u(0)=u_{0} .
$$

Assume both that $F: \mathbb{R}^{n} \rightarrow \mathbb{R}^{n}$ is continuous and that there is a unique solution $u\left(t ; u_{0}\right)$ through any initial condition $u_{0}$ (for $t \in \mathbb{R}$ ). (So, for example, $F$ could be only Hölder continuous,

$$
|F(u)-F(v)| \leq C|u-v|^{\theta}, \quad 0<\theta<1,
$$

instead of Lipschitz ((1.2) with $\theta=1)$ which is the usual condition used to guarantee uniqueness.)

Denote the solution map which takes $u_{0}$ to $u\left(t ; u_{0}\right)$ by $\varphi(t)$, so that

$$
\varphi(t) u_{0}=u\left(t ; u_{0}\right)
$$

this will simplify the notation throughout the paper.

The two assumptions above (that $F$ is continuous and that solutions are unique) guarantee (Hartman 1964, Chapter V, theorem 2.1) that the solution $u\left(t ; u_{0}\right)$ depends continuously on the initial condition $u_{0}$, as well as $t$. Note that if $X$ is a compact set, this means that given $\epsilon>0$ and $T>0$, there is a $\delta>0$ such that

$$
u_{0}, v_{0} \in X \quad \text { and } \quad\left|u_{0}-v_{0}\right| \leq \delta
$$


implies

$$
\sup _{t \in[0, T]}\left|\varphi(t) u_{0}-\varphi(t) v_{0}\right| \leq \epsilon
$$

To confine the asymptotic behaviour of (1.1) to some bounded region, it will be assumed that the equation is dissipative, so that there is a bounded set $B$ which absorbs any bounded set $X$ in a finite time,

$$
\varphi(t) X \subseteq B \quad \text { for all } t \geq t_{0}(X)
$$

(This means that $\varphi(t) u_{0} \in B$ once $t \geq t_{0}(X)$, for all $u_{0} \in X$.)

This assumption on equation (1.1) is sufficient (Billotti and LaSalle 1971) to show that there is a compact set $\mathcal{A}$ which is invariant,

$$
\text { if } \quad u_{0} \in \mathcal{A} \quad \text { then } \quad \varphi(t) u_{0} \in \mathcal{A} \quad \text { for all } \quad t \in \mathbb{R} \text {, }
$$

and attracting, so that for any $u_{0}$,

$$
\operatorname{dist}\left(\varphi(t) u_{0}, \mathcal{A}\right) \rightarrow 0 \quad \text { as } \quad t \rightarrow \infty
$$

where

$$
\operatorname{dist}(x, Y)=\inf _{y \in Y}|x-y| \text {. }
$$

(This attraction is actually uniform over $u_{0}$ in any bounded set $X$.)

The set $\mathcal{A}$ is termed the "global attractor", and has been the object of much research, particularly in the context of partial differential equations (see Temam (1988) for many examples). The dynamics on the set $\mathcal{A}$ determines all the asymptotic behaviour of the system, and this is formalised in the following proposition, taken from Langa and Robinson (1996). In essence, the solutions of (1.1) follow (portions of) trajectories on $\mathcal{A}$ closely for long time intervals once they get near enough to $\mathcal{A}$. 
Proposition 1.1. Given a solution $u(t)$ of (1.1), a $T>0$ and and an $\epsilon>0$, there exists a time $\tau>0$ and a sequence of points $\left\{v_{n}\right\}_{n=0}^{\infty}$, with $v_{n} \in \mathcal{A}$, such that

$$
\left|u(\tau+n T+t)-\varphi(t) v_{n}\right| \leq \epsilon \quad 0 \leq t \leq T
$$

and

$$
\left|v_{n+1}-\varphi(T) v_{n}\right| \leq 2 \epsilon \text {. }
$$

The orbit segments $\left\{\varphi(t) v_{n}: 0 \leq t \leq T\right\}$ form a $(T, 2 \epsilon)$ pseudo-trajectory of (1.1) - this means precisely that each portion satisfies (1.1) and that (1.7) holds. Thus one can reformulate proposition 1.1 by saying that, after a transient time $\tau$, any trajectory of (1.1) is determined to within $\epsilon$ - or " $\epsilon$-shadowed" - by a $(T, 2 \epsilon)$ pseudo-trajectory of (1.1) lying entirely on the attractor.

This result guarantees that an understanding of the dynamics on the attractor provides an understanding of the dynamics of the full equation. The aim is to approximate the dynamics restricted to $\mathcal{A}$ using the trajectories of a threedimensional flow.

\section{The main result and sketch proof}

The main result of this paper guarantees that any solution lying on the global attractor of the original $n$-dimensional equation can be approximated arbitrarily closely (to within any $\epsilon>0$ ), and for an arbitrarily long time, by a solution of a three-dimensional differential equation $d x / d t=f(x)$, where $f$ is a Lipschitz function,

$$
|f(x)-f(y)| \leq L|x-y| \quad \text { for some } \quad L>0 .
$$

This smoothness of $f$ guarantees that the equation has unique solutions (see Hartman (1964), for example).

A formal statement is as follows: 
Theorem 2.1. Choose $T>0$ and $\epsilon>0$. Then there exist functions

$$
f: \mathbb{R}^{3} \rightarrow \mathbb{R}^{3} \quad \text { and } \quad \Phi: \mathbb{R}^{3} \rightarrow \mathbb{R}^{n}
$$

Lipschitz and Hölder continuous respectively, such that given any initial condition $u_{0} \in \mathcal{A}$, there is a solution $x(t)$ of

$$
d x / d t=f(x)
$$

with

$$
\left|\Phi(x(t))-\varphi(t) u_{0}\right| \leq \epsilon \quad \text { for all } \quad 0 \leq t \leq T
$$

Note that although trajectories are only approximated for a finite length of time $T$, this still serves to determine the asymptotic behaviour, as the portions of solution approximated lie on the global attractor: one has already taken the limit " $t \rightarrow \infty$ " to obtain the set $\mathcal{A}$.

A simple induction argument then shows that any solution on $\mathcal{A}$ can be approximated for all time by changing from one trajectory of $d x / d t=f(x)$ to another after every time interval $T$. The "jumps" in the trajectories of the threedimensional system at these times are also no larger than $2 \epsilon$ :

Corollary 2.2. If $u_{0} \in \mathcal{A}$ and $u(t)=u\left(t ; u_{0}\right)$, then there exist solutions $x_{j}(t)$ of

$$
d x / d t=f(x) \quad x_{0}=x_{j}(0),
$$

such that

$$
\left|\Phi\left(x_{j}(t)\right)-u(j T+t)\right| \leq \epsilon \quad 0 \leq t \leq T \quad j=0,1,2, \ldots
$$

Furthermore,

$$
\left|x_{j}(T)-x_{j+1}(0)\right| \leq 2 \epsilon .
$$


The corollary embodies important extra information on the nature of the system obtained in theorem 2.1. As in proposition 1.1, corollary 2.2 says that there is a $(T, 2 \epsilon)$ pseudo-orbit of $d x / d t=f(x)$ whose image under $\Phi \epsilon$-shadows any trajectory $u(t)$ lying on the attractor $\mathcal{A}$. Note that combining corollary 2.2 with proposition 1.1 shows that any solution of the original equation can be shadowed by a pseudo-orbit of $d x / d t=f(x)$ after some transient time.

The use of the global attractor, and the consequent discounting of transient behaviour, is not central to the argument. One can in fact replace the global attractor $\mathcal{A}$ in theorem 2.1 (and corollary 2.2) with any compact set $K$. Thus the solutions through any bounded set of initial conditions (which are compact in a finite-dimensional space like $\mathbb{R}^{n}$ ) can be shadowed by the pseudo-orbit of a three-dimensional system.

Such a result is a standard way of saying that two systems are, in some sense, "close" to each other (e.g. Mielke (1997) uses such a notion to show that the effects of boundary conditions in the complex Ginzburg-Landau equation are small within the interior of large domains). Indeed, it can be argued that the approximation given by a standard numerical scheme is no better than that provided by theorem 2.1 and corollary 2.2 .

Suppose that $\xi(t)$ is the approximation to a solution $u(t)$ on $\mathcal{A}$ generated by a numerical method with time-step $\Delta t$, say. Then standard error estimates are of the form (see Stuart and Humphries (1996), for example)

$$
|u(t)-\xi(t)| \leq C(t) \Delta t^{r}
$$

where $C(t)$ is an increasing function of $t$ with $C(0)=0$. Thus, with a fixed timestep, the solution can only be approximated to within an $\epsilon$ error over some fixed 
time interval $T$,

$$
|u(t)-\xi(t)| \leq \epsilon \quad \text { for all } \quad t \in[0, T]
$$

as in (2.1). To continue the approximation to within the desired accuracy it is necessary either to start the numerical solution again from another initial condition, or to refine the time-step. Starting with another initial condition is the approach adopted in corollary 2.2, whereas choosing a smaller timestep corresponds to obtaining a different $f(x)$ in theorem 2.1.

A sketch of the proof of theorem 2.1 should make the argument much clearer. Seeking only an approximation of the trajectories gives much freedom, and this will be exploited to the full. Furthermore, taking only a finite time interval is central to the construction.

First, the compactness of $\mathcal{A}$ allows the dynamics on the set to be shadowed (to within $\epsilon$ on $[0, T]$ ) by the trajectories through a finite set of initial conditions. These trajectories are each one-dimensional objects, and so the whole collection of them is also one-dimensional. This is the key observation: that the dynamics on $\mathcal{A}$ are determined (to within approximation) by a one-dimensional set of trajectories.

A result of Mañé (1981), recently improved by Foias and Olson (1996), guarantees that any $d$-dimensional set can be embedded into $\mathbb{R}^{[2 d+1]}$, where $[x]$ denotes the smallest integer greater than or equal to $x$. Thus a 1-dimensional set can be embedded into $\mathbb{R}^{3}$. Hence there is a natural way to construct a three-dimensional system which gives the dynamics of these specially selected trajectories on $\mathcal{A}$ exactly.

However, this first system of three differential equations is not smooth enough to guarantee uniqueness. So the final step is to construct a (Lipschitz) smooth system which has solutions near those which were embedded from $\mathcal{A}$. These can 
be "lifted back" into $\mathbb{R}^{n}$ to approximate the special trajectories on $\mathcal{A}$, and so all trajectories on $\mathcal{A}$.

The next section gives the definition of fractal dimension, and the embedding theorem referred to above, along with a theorem on the extension of continuous functions which will be used in the proof of theorem 2.1 .

\section{The embedding and extension theorems}

To formulate the embedding theorem which is fundamental in the proof of theorem 2.1, it is necessary to introduce the concept of the fractal dimension.

The fractal dimension is the strongest commonly used measure of dimension (for a full discussion of fractional measures of dimension see Falconer 1990), and is based an approximation of the $d$-dimensional volume of a compact set $X$ by balls of fixed radius.

Given $\epsilon>0$, let $N(\epsilon, X)$ denote the number of balls of radius $\epsilon$ necessary to cover $X$. Then the fractal dimension of $X, d_{F}(X)$, is defined as

$$
d_{F}(X)=\liminf _{\epsilon \rightarrow 0} \frac{\log N(\epsilon, X)}{\log (1 / \epsilon)} .
$$

Two important properties of the fractal dimension which will be needed later are its behaviour under unions,

$$
d_{F}(X \cup Y)=\max \left(d_{F}(X), d_{F}(Y)\right)
$$

and under Lipschitz maps: if $\psi$ is a Lipschitz function, then

$$
d_{F}(\psi(X)) \leq d_{F}(X)
$$

Proofs are given in Falconer (1990). 
In 1981 Mañé showed, using an argument based on the Baire category theorem, that "most" orthogonal projections of a set with a finite fractal dimension $d$ onto subspaces of dimension $[2 d+1]$ are injective. This was improved by Eden et al. (1994), who give a constructive proof and provide some continuity properties of the inverse of this projection when $X$ is a subset of $\mathbb{R}^{n}$. The best current result is due to Foias and Olson (1996), who extend this to the case when $X$ is a subset of a Hilbert space $H$ :

Theorem 3.1 (Mañé; Foias and Olson). Let $X$ be a compact subset of a Hilbert space $H$ with $d_{F}(X) \leq d$. Then there exists an orthogonal projection $P$ onto a subspace of dimension $[2 d+1]$, which is injective on $X$, and whose inverse is Hölder continuous on $P X$,

$$
\left|P^{-1} x-P^{-1} y\right| \leq C|x-y|^{\theta} \quad x, y \in P X
$$

for some $C>0$ and $0<\theta<1$.

(In fact the theorem is stronger, asserting that such projections are dense in the set of all orthogonal projections onto subspaces of dimension $[2 d+1]$.)

The following extension theorem guarantees that any continuous function on a closed subset of $\mathbb{R}^{m}$ can be extended to the whole of $\mathbb{R}^{m}$, without losing any nice continuity properties of the original function. The proof can be found in Stein (1970, corollary of theorem VI.3).

Theorem 3.2 (Stein). Let $X$ be a closed subset of $\mathbb{R}^{m}, H$ a Hilbert space, and $f: X \rightarrow H$ a continuous function. Then there is a continuous extension $E_{X}(f)$ of $f$ to the whole of $\mathbb{R}^{m}, E_{X}(f): \mathbb{R}^{m} \rightarrow H$, and this is linear and continuous in $f$ (in the sup norm). Furthermore there exists a $K=K(m)$ such that if

$$
|f(x)-f(y)| \leq C|x-y|^{\theta}
$$


for some $0<\theta \leq 1$, then

$$
\left\|E_{X}(f)\right\|_{\infty} \leq \sup _{x \in X}|f(x)| \quad \text { and } \quad\left|E_{X}(f)(x)-E_{X}(f)(y)\right| \leq K C|x-y|^{\theta} .
$$

\section{Proof of the theorem and corollary}

Recall that the aim is to find functions

$$
f: \mathbb{R}^{3} \rightarrow \mathbb{R}^{3} \quad \text { and } \quad \Phi: \mathbb{R}^{3} \rightarrow \mathbb{R}^{n}
$$

Lipschitz and Hölder continuous respectively, so that every solution $u(t)$ on $\mathcal{A}$ can be closely approximated by some solution $x(t)$ of $d x / d t=f(x)$ over a fixed time interval $[0, T]$

$$
|\Phi(x(t))-u(t)| \leq \epsilon \quad \text { for all } \quad 0 \leq t \leq T
$$

Observe that the proof of the theorem is identical if $\mathcal{A}$ is replaced by an arbitrary compact set $K$.

Proof. The first step of the proof is to use the compactness of $\mathcal{A}$ and the continuous dependence on initial conditions to pick out a finite set of initial conditions which "cover" the global attractor to within $\epsilon$ on $[0, T]$.

Since $\mathcal{A}$ is compact, the solutions $u\left(t ; u_{0}\right)$ depend continuously on $u_{0}$ uniformly over $\mathcal{A}$ (which is (1.3)). So given $\epsilon$ and $T$ as in the statement of the theorem, there exists a $\delta>0$ such that

$$
\left|u_{0}-v_{0}\right| \leq \delta \quad \Rightarrow \quad\left|\varphi(t) u_{0}-\varphi(t) v_{0}\right| \leq \epsilon / 2 \quad \text { for all } 0 \leq t \leq T
$$

Now take a cover of $\mathcal{A}$ by balls of radius $\delta$. Since $\mathcal{A}$ is compact there exists a finite sub-cover. Denote the centres of the balls in this finite sub-cover by $\left\{u_{k}\right\}$, where $k$ runs between 1 and $\kappa \in \mathbb{N}$. Then given a point $u_{0} \in \mathcal{A}$, there is a $u_{k}$ with

$$
\left|u_{0}-u_{k}\right| \leq \delta
$$


(4.2) ensures that the trajectory $u_{k}(t)=u\left(t ; u_{k}\right)$ differs from $u(t)=u\left(t ; u_{0}\right)$ on $[0, T]$ by at most $\epsilon / 2$.

Now consider the trajectory through $u_{k}$ between $t=0$ and $t=T$,

$$
\tau_{k}=\bigcup_{0 \leq t \leq T} \varphi(t) u_{k}
$$

Since $F$ is continuous and $\mathcal{A}$ is compact, $F$ is bounded on $\mathcal{A}$. This implies that the solution $u\left(t ; u_{0}\right)$ is Lipschitz in $t$,

$$
\left|u\left(t ; u_{0}\right)-u\left(s ; u_{0}\right)\right| \leq M|t-s|
$$

for some $M$. Thus $\tau_{k}$ is the Lipschitz image of the set $[0, T]$, and so by $(3.2)$ the fractal dimension of $\tau_{k}$ is at most 1 ,

$$
d_{F}\left(\tau_{k}\right) \leq 1
$$

Now let $\mathcal{T}$ be the union (over $k$ ) of these sets,

$$
\mathcal{T}=\cup_{k} \tau_{k}
$$

As a finite union of sets of fractal dimension at most 1, (3.1) ensures that $\mathcal{T}$ itself has fractal dimension at most 1 ,

$$
d_{F}(\mathcal{T}) \leq 1
$$

This is the one-dimensional set that gives approximations to all the trajectories on $\mathcal{A}$.

When constructing the differential equation on $\mathbb{R}^{3}$ to make the nonlinear term Lipschitz, it will be important that the embedded sections of trajectory are 
separated from each other. To make sure of this it is necessary to rewrite $\mathcal{T}$ as the union of disjoint portions of trajectory.

Note that if, for some $k \neq j$, two portions of trajectory intersect,

$$
\tau_{k} \cap \tau_{j} \neq \emptyset
$$

then, since solutions on $\mathcal{A}$ are unique in both forwards and backwards time, together they form a larger portion of one trajectory

$$
\tau_{k} \cup \tau_{j}=\bigcup_{0 \leq t \leq \tilde{T}} \varphi(t) v
$$

where $T \leq \tilde{T} \leq 2 T$, and $v$ is either $u_{k}$ or $u_{j}$.

Thus $\mathcal{T}$ can be written as the union of disjoint portions of trajectories

$$
\tilde{\tau}_{j}=\bigcup_{0 \leq t \leq T_{j}} \varphi(t) \tilde{u}_{j}
$$

with $\tilde{u}_{j}=u_{k}$ for some $k$, and $T \leq T_{j} \leq \kappa T$ :

$$
\mathcal{T}=\cup_{j} \tilde{\tau}_{j}
$$

The embedding theorem 3.1 guarantees the existence of an orthogonal projection $P: \mathbb{R}^{n} \rightarrow \mathbb{R}^{3}$, injective on $\mathcal{T}$ and with Hölder continuous inverse on $P \mathcal{T}$ (3.3). Clearly one can identify $P \mathbb{R}^{n}$ with $\mathbb{R}^{3}$ using three orthogonal directions in $P \mathbb{R}^{n}$ as a basis.

The inverse of $P$, which is well-defined only on $P \mathcal{T}\left(P^{-1} x\right.$ is the unique point $u \in \mathcal{T}$ with $P u=x$ ), can be extended to a function $\Phi$ defined on the whole of $\mathbb{R}^{3}$ and such that $P \circ \Phi=$ id. Indeed, rather than extending $P^{-1}$ itself with theorem 3.2 , extend

$$
\phi^{\perp}(x)=P^{-1} x-x \quad \phi^{\perp}: P \mathcal{T} \rightarrow\left(P \mathbb{R}^{n}\right)^{\perp}
$$


to a function $\Phi^{\perp}: P \mathbb{R}^{n} \rightarrow\left(P \mathbb{R}^{n}\right)^{\perp}$. Finally, set

$$
\Phi(x)=x+\Phi^{\perp}(x) .
$$

Clearly, $P \circ \Phi=\mathrm{id}$, and since $P^{-1}$ is Hölder on $P \mathcal{T}$ (by (3.3)), so is $\phi^{\perp}$; equation (3.4) of the extension theorem gives Hölder continuity of $\Phi^{\perp}$ and so of $\Phi$.

Note that if $\epsilon$ is smaller or $T$ is larger, the trajectories in $\mathcal{T}$ cover $\mathcal{A}$ more densely. Thus the image of $\mathcal{T}$ under $P$ will be more tightly packed in $\mathbb{R}^{3}$, and hence one would expect the inverse of $P$ to be "less continuous" (e.g. perhaps with smaller Hölder exponent $\theta$ in $(3.3))$.

It is now possible to write down a three-dimensional differential equation which reproduces exactly the trajectories projected into $\mathbb{R}^{3}$ from $\mathcal{T}$. The equation for the projection $x(t)$ of one of the trajectories $u(t)$ from $\mathcal{T}(x(t)=P u(t))$ is

$$
\frac{d x}{d t}=\frac{d}{d t}(P u)=P \frac{d u}{d t}=P F(u)=P F\left(P^{-1} u\right) \quad x \in P \mathcal{T}
$$

Since $P, F$, and $P^{-1}$ are all continuous, the function $g$ defined on $P \mathcal{T}$ by

$$
g(x) \equiv P F\left(P^{-1} x\right)
$$

is continuous from $\mathbb{R}^{3}$ into itself. The extension theorem 3.2 guarantees that $g$ can be extended to a continuous function $g: \mathbb{R}^{3} \rightarrow \mathbb{R}^{3}$. However, since $g$ is not Lipschitz continuous, one cannot guarantee that the solutions of $d x / d t=g(x)$ are unique.

To remedy this, the following construction produces a Lipschitz vector field, one solution of which stays close to each of the projected solutions in $P \mathcal{T}$. Since each $P \tilde{\tau}_{j}$ is compact and

$$
P \tilde{\tau}_{j} \cap P \tilde{\tau}_{k}=\emptyset \quad \text { for } \quad j \neq k
$$


there is some uniform separation between the images of the $\tau_{k}$, i.e. there exists a $\sigma$ such that

$$
|x-y| \geq 3 \sigma \quad x \in P \tilde{\tau}_{j}, y \in P \tilde{\tau}_{k} \quad j \neq k .
$$

It follows that the $\sigma$-neighbourhoods $N_{j}$ of each of the $P \tilde{\tau}_{j}$,

$$
N_{j}=\left\{x+y: x \in P \tilde{\tau}_{j},|y| \leq \sigma\right\}
$$

are separated by $\sigma$,

$$
|x-y| \geq \sigma \quad x \in N_{j}, y \in N_{k} \quad j \neq k .
$$

By restricting attention to each of these neighbourhoods in turn, the problem reduces to finding a suitable vector field to approximate one portion of trajectory $x(t)$, that lying within $N_{j}$, say

$$
x(t)=P u\left(t ; \tilde{u}_{j}\right) .
$$

To this end, choose $\eta \leq \sigma$ small enough that

$$
|x-y| \leq \eta \quad \Rightarrow \quad|\Phi(x)-\Phi(y)| \leq \epsilon / 2 .
$$

This allows an error of $\eta$ between the new trajectory and the old one while still ensuring that the images of the trajectories in $\mathbb{R}^{n}$ (under $\Phi$ ) remain close.

Essentially, $x(t)$ will be approximated by a piecewise linear trajectory; this will then be adjusted slightly to obtain a Lipschitz continuous vector field. First, split the time interval $[0, T]$ into pieces $\left[t_{i}, t_{i+1}\right]$, where $t_{0}=0$ and $t_{i+1}$ is the greatest time such that

$$
\left|x(t)-x\left(t_{i}\right)\right| \leq \eta / 4 \quad \text { for all } \quad t \in\left[t_{i}, t_{i+1}\right] .
$$


There are a finite number of such pieces, since the Lipschitz property of solutions (4.4) guarantees that $t_{i+1}-t_{i} \geq \eta / M$.

The piecewise linear trajectory $x_{l}^{1}(t)$ which joins $x\left(t_{i}\right)$ to $x\left(t_{i+1}\right)$, and has

$$
x_{l}^{1}\left((1-\theta) t_{i}+\theta t_{i+1}\right)=(1-\theta) x\left(t_{i}\right)+\theta x\left(t_{i+1}\right)
$$

clearly satisfies

$$
\left|x_{l}^{1}(t)-x(t)\right| \leq \eta / 4
$$

However, this first linear trajectory may have self-intersections. To remedy this, define $x_{i} \approx x\left(t_{i}\right)$ (strictly it is only necessary to ensure that $\left|x_{i}-x\left(t_{i}\right)\right| \leq \eta / 4$ ) such that the piecewise linear trajectory $x_{l}(t)$, joining the $x_{i}$ as in (4.7), has no self-intersections and satisfies

$$
\left|x_{l}(t)-x(t)\right| \leq \eta / 2
$$

(and consequently $x_{l}$ still lies entirely within $N_{j}$ ).

The vector field

$$
f\left((1-\theta) x_{i}+\theta x_{i+1}\right)=f_{i}=\frac{x_{i+1}-x_{i}}{t_{i+1}-t_{i}}, \quad 0 \leq \theta<1,
$$

constant along each linear portion of trajectory, gives the solution $x_{l}(t)$. However, it is not even continuous, as it has sharp "corners" at each $x_{i}$.

To "round off the corners", define $\delta_{i}$ such that the trajectory does not return to a $2 \delta_{i}$ neighbourhood of $x_{i}$,

$$
\left|x_{l}(t)-x_{i}\right| \geq 2 \delta_{i} \quad \text { for all } \quad t \geq t_{i+1}
$$

The idea is then to adjust the linear trajectory (and so $f$ ) within

$$
S_{i}=\left\{\left|x-x_{i}\right| \leq \delta_{i}\right\}
$$


such that $f$ is Lipschitz on its domain of definition (precisely the trajectory itself), and that it takes a time $T$,

$$
T=\frac{\delta}{\left|f_{i}\right|}+\frac{\delta}{\left|f_{i+1}\right|}
$$

from the point of ingress to $S_{i}, y_{i}^{-}$, to its point of egress from $S_{i}, y_{i}^{+}$. This ensures that the time taken to reach $y_{i}^{+}$using the adjusted trajectory is the same as it was with $x_{l}(t)$.

To show that this is possible, consider first the case where $y^{-}$is opposite $y^{+}$; then the problem reduces to constructing a one-dimensional Lipschitz $g$ on $[-\delta, \delta]$ with

$$
g(-\delta)=g_{1} \quad g(\delta)=g_{2} \quad g_{1}=\left|f_{1}\right|, g_{2}=\left|f_{2}\right|,
$$

such that the solution of

$$
d y / d t=g(y) \quad y(0)=-\delta
$$

has $y(T)=\delta$. A family of possible functions $g$, parametrised by $g^{*}$, is

$$
g(y)= \begin{cases}\left(g^{*}-g_{1}\right) \frac{y+\delta}{\delta}+g_{1} & -\delta \leq y \leq 0 \\ \left(g_{2}-g^{*}\right) \frac{y}{\delta}+g_{2} & 0 \leq y \leq \delta\end{cases}
$$

for which the time taken from $y=-\delta$ to $y=\delta$ is

$$
T\left(g^{*}\right)=\delta\left(\frac{\ln g_{1}-\ln g^{*}}{g_{1}-g^{*}}+\frac{\ln g_{2}-\ln g^{*}}{g_{2}-g^{*}}\right) .
$$

Assume that $g_{1}>g_{2}$; then it is straightforward to show that $T\left(g_{1}\right)<T$, and $T\left(g_{2}\right)>T$, so there is some value of $g^{*}\left(g_{2}<g^{*}<g_{1}\right)$ that gives the correct value of $T$ as in (4.8).

Now take the one-dimensional trajectory from (4.9) and map it onto a smooth orbit joining $y^{-}$and $y^{+}$, using a mapping $\psi$ with the following properties:

(i) $\psi:[0,2 \delta] \rightarrow S_{i}$, 
(ii) $\psi$ is $C^{2}$,

(iii) $\psi^{-1}: \psi([0,2 \delta]) \rightarrow[0,2 \delta]$ is Lipschitz,

(iv) $\psi(0)=y^{-}, \psi(2 \delta)=y^{+}$, and

(v) $\nabla \psi(0)=-\mathbf{e}_{r}, \nabla \psi(2 \delta)=\mathbf{e}_{r}$, where $\mathbf{e}_{r}$ is the outward normal to $S_{i}$.

Condition (i) ensures that the orbit remains within $S_{i}$; conditions (ii) and (iii) that the vector field on the orbit is Lipschitz: if $x=\psi(y)$, then

$$
d x / d t=f(x)=\nabla \psi(y) g(y)
$$

where $g$ is given by (4.10) and $y=\psi^{-1}(x)$. Since $g$ is Lipschitz, so is $f$. (iv) ensures that the orbit joins $y^{-}$to $y^{+}$, and $(\mathrm{v})$ that the derivatives match up there.

Thus, defining $x_{a}$ to be equal to $x_{l}$ outside the spheres $S_{i}$, and using the above construction within each sphere, one obtains a trajectory $x_{a}$ (within $N_{j}$ ) satisfying

$$
d x_{a} / d t=f\left(x_{a}\right)
$$

$f$ is defined only on $x_{a}$, but Lipschitz over its domain of definition. Applying this construction to each $\tau_{j}$ gives an $f$ such that the unique solution $x(t)$ of $d x / d t=$ $f(x)$ with initial condition $x(0)=P \tilde{u}_{j}$ stays within $\eta$ of $P u\left(t ; \tilde{u}_{j}\right)$ on $\left[0, \tilde{T}_{j}\right]$,

$$
\left|x(t)-P u\left(t ; \tilde{u}_{j}\right)\right| \leq \eta \quad 0 \leq t \leq \tilde{T}_{j}
$$

Since $\Phi \circ P=$ id on $\mathcal{T},(4.6)$ shows that the image of $x(t)$ under $\Phi$ stays within $\epsilon / 2$ of $u\left(t ; \tilde{u}_{j}\right)$,

$$
\left|\Phi(x(t))-u\left(t ; \tilde{u}_{j}\right)\right| \leq \epsilon / 2 \quad 0 \leq t \leq \tilde{T}_{j}
$$

It remains is to combine the definitions of the function $f$ on the disjoint trajectories $x_{a}^{j}$ to give a Lipschitz function on the whole of $\mathbb{R}^{3}$. This will be 
straightforward using the extension theorem 3.2, provided that $f$ is Lipschitz on $\cup_{j} x_{a}^{j}$.

Since $f$ is Lipschitz on each trajectory $x_{a}^{j}$, there is a constant $K_{1}$ such that if $x$ and $y$ lie on the same $x_{a}^{j}$ (for any $j$ )

$$
|f(x)-f(y)| \leq K_{1}|x-y| \quad x, y \in x_{a}^{j} .
$$

If $x \in x_{a}^{j}$ and $y \in x_{a}^{k}$ with $j \neq k$, then, since $f$ is bounded on each trajectory,

$$
|f(x)-f(y)| \leq 2\|f\|_{\infty} \leq \frac{2\|f\|_{\infty}}{\sigma}|x-y| \equiv K_{2}|x-y|
$$

using (4.5). (If the trajectories are closely packed, $\sigma$ is small and the Lipschitz constant of $f$ will be large.)

Thus $f$ is Lipschitz on the closed set $\cup_{j} x_{a}^{j}$, and so can be extended to a function on the whole of $\mathbb{R}^{3}$ that is globally Lipschitz, using theorem 3.2.

Finally, to prove the theorem, take a solution $u\left(t ; u_{0}\right)$ with $u_{0} \in \mathcal{A}$. Then by (4.3), there is a $u_{k}$ with $\left|u_{k}-u_{0}\right| \leq \delta$, and so a solution $v(t)=\varphi(t) u_{k}$ in $\mathcal{T}$ which satisfies

$$
\left|v(t)-\varphi(t) u_{0}\right| \leq \epsilon / 2 \quad \text { for all } \quad 0 \leq t \leq T \text {. }
$$

Since there is a solution of

$$
d x / d t=f(x)
$$

which satisfies (from (4.11))

$$
|\Phi(x(t))-v(t)| \leq \epsilon / 2
$$

one can combine this with (4.12) to give

$$
\left|\Phi(x(t))-\varphi(t) u_{0}\right| \leq \epsilon \quad 0 \leq t \leq T,
$$


which is (4.1) and the theorem is proved.

Notice that as the set $\mathcal{T}$ consists of a finite number of $\operatorname{arcs}$ in $\mathbb{R}^{n}$, it would be possible to embed these arcs one-by-one into the real line, using some embedding $\mathcal{P}$ continuous on $\mathcal{T}$. One could then follow the same construction as above (although $\Phi=\mathcal{P}^{-1}$ would not necessarily be Hölder continuous) to obtain an essentially vacuous result. However, such an embedding would take points which were close in $\mathcal{A}$, but on different orbit segments $\tau_{k}$, and send them to points far apart in $\mathbb{R}$. Since the chosen embedding is an orthogonal projection

$$
|P(u-v)| \leq|u-v|
$$

and points which are close cannot be separated under $P$. It is this property of projections that allows the proof of the corollary, and ensures that the content of theorem 2.1 is not "vacuous" like the above construction on $\mathbb{R}$.

Approximation of a trajectory through $u_{0}$ is possible for all time by skipping from one trajectory of $d x / d t=f(x)$ to the other. The main content of corollary 2.2 is that the "jumps" are not too large,

$$
\left|x_{j}(T)-x_{j+1}(0)\right| \leq 2 \epsilon .
$$

Proof. For $j=0$ this is exactly theorem 2.1. Now, at the maximum time of approximation $T$, the shadowing result implies that

$$
\left|\Phi\left(x_{0}(T)\right)-u(T)\right| \leq \epsilon
$$

Since $P$ is a projection, $|P w| \leq|w|$, and as $P \circ \Phi=\mathrm{id}$,

$$
\left|x_{0}(T)-P u(T)\right| \leq \epsilon
$$


also.

Now apply theorem 2.1 to the trajectory $v$ with initial condition $u(T), v(t)=$ $v(t ; u(T)) .(2.1)$ then shows that there is a solution $x_{1}(t)$ of $d x / d t=f(x)$ with

$$
\left|\Phi\left(x_{1}(0)\right)-v(0)\right|=\left|\Phi\left(x_{1}(0)\right)-u(T)\right| \leq \epsilon .
$$

Once again, applying $P$ gives

$$
\left|x_{1}(0)-P u(T)\right| \leq \epsilon
$$

The triangle inequality gives

$$
\left|x_{0}(T)-x_{1}(0)\right| \leq 2 \epsilon
$$

and the result follows by induction.

Note that, from the construction, $f$ depends on both $\epsilon$ and $T$. As $\epsilon$ decreases and $T$ increases, the Lipschitz constant of $f$ will become ever larger, and the continuity properties of $f$ degrade in the limit of small $\epsilon$ and large $T$.

If one wishes to extend the corollary to solutions through initial conditions in an arbitrary compact set $K$, some care is need, since $\varphi(T) K \neq K$. To use the same inductive argument as above, it is necessary to ensure that

$$
\bigcup_{0 \leq t \leq \infty} \varphi(t) K
$$

is contained in a compact set $\mathcal{K}$. However, this follows from the dissipativity property (1.4), since

$$
\bigcup_{0 \leq t \leq t_{0}(K)} \varphi(t) K
$$

is closed and bounded, and $\varphi(t) K \subset B$ for all $t \geq t_{0}(K)$. For $u_{0} \in K$ theorem 2.1 (with $\mathcal{A}$ replaced by $\mathcal{K}$ ) can then be applied repeatedly as before. 


\section{Dissipative partial differential equations}

This dramatic reduction in the dimension of the problem is also possible for the global attractors of partial differential equations. Indeed, it was in this context (Robinson 1997) that the result was first derived as something of an aside.

In many cases the solutions of a partial differential equation can be used to define a dynamical system on some appropriate infinite-dimensional phase space. For example, consider the one-dimensional Kuramoto-Sivashinksy equation with periodic boundary conditions,

$$
u_{t}+u_{x x x x}+u_{x x}+u u_{x}=0 \quad u(x+L, t)=u(x, t),
$$

a model for instabilities in films and interfaces (see Nicolaenko et al. (1985) for further mathematical details). One can show that if the initial condition is odd then the solution is odd for all time; so, restricting to odd solutions, expand $u(x, t)$ as a Fourier sine series,

$$
u(x, t)=\sum_{1}^{\infty} y_{n}(t) \sin \frac{2 n \pi x}{L} .
$$

Substituting this expansion into (5.1) and equating coefficients gives an infinite set of coupled ordinary differential equations for the $\left\{y_{n}\right\}$,

$$
\frac{d y_{n}}{d t}+\lambda_{n} y_{n}-\frac{n \pi}{L} \sum_{j=1}^{\infty} y_{j} y_{n+j}+\frac{\pi}{L} \sum_{k=1}^{n-1} k y_{k} y_{n-k}=0
$$

with

$$
\lambda_{n}=\left(\frac{2 n \pi}{L}\right)^{4}-\left(\frac{2 n \pi}{L}\right)^{2}
$$

the original partial differential equation corresponds to an infinite-dimensional system. The condition that the $l^{2}$ norm of the infinite vector $\left(y_{1}, y_{2}, \ldots\right)$ is finite,

$$
\sum_{j=1}^{\infty}\left|y_{j}\right|^{2}<\infty
$$


is equivalent, by Parseval's theorem, to $u(x, t)$ being square integrable,

$$
\int_{0}^{L}|u(x, t)|^{2} d x<\infty
$$

i.e. the $L^{2}$ norm of $u(x, t)$ is finite. Indeed, the appropriate phase space for $(5.1)$ is $L^{2}(0, L)$; i.e. if $u_{0} \in L^{2}(0, L)$ then there is a unique solution $u(x, t)$, which exists for all positive time and is in $L^{2}(0, L)$ for all $t \geq 0$.

A solution of (5.2) corresponds to a weak solution of (5.1), i.e. a solution of the equation

$$
\left(u_{t}, v\right)+\left(u_{x x x x}, v\right)+\left(u_{x x}, v\right)+\left(u u_{x}, v\right)=0
$$

where $v$ is a smooth $L$-periodic function, and $(\cdot, \cdot)$ is the standard $L^{2}$ inner product,

$$
(u, v)=\int_{0}^{L} u(x) v(x) d x .
$$

This allows generalisation of the notion of a "solution" of (5.1), since one can integrate by parts to obtain

$$
\left(u_{t}, v\right)+\left(u, v_{x x x x}\right)+\left(u, v_{x x}\right)+\frac{1}{2}\left(u^{2}, v_{x}\right)=0
$$

which still makes sense even if $u$ is not differentiable. If a solution of (5.3) is sufficiently smooth, then one can show that it is a classical solution of (5.1)

To enable a general treatment, assume that the given partial differential equation generates a semiflow on a Hilbert space $\mathcal{H}$ (which is usually $L^{2}$ or some related space), so that given an initial condition $u_{0} \in \mathcal{H}$ the solution $u\left(t ; u_{0}\right)$ exists and is unique for all $t \geq 0$ (in general, one does not expect backwards existence $(t<0$ ) for dissipative PDEs).

For the existence of a global attractor, the conditions of section 1 need to be strengthened, since now a bounded set is no longer necessarily compact (recall 
that a metric space is finite dimensional iff its unit ball is compact). Under the condition that the absorbing set $B$ in (1.4) is compact (this can be weakened - see Hale (1988) or Temam (1988) for other more general formulations of the result) there is a compact set $\mathcal{A}$, which is attracting and invariant as before $((1.5)$ and $(1.6))$.

A careful examination of the proof of theorem 2.1 shows that for application to infinite-dimensional systems the following hypotheses need to be checked:

(i) The equation has a global attractor $\mathcal{A}$ which is compact in $\mathcal{H}$;

(ii) Solutions must have the "backwards uniqueness" property; namely that if $u\left(t ; u_{0}\right)=u\left(t ; v_{0}\right)$ for some $t>0$, then $u_{0}=v_{0} ;$

(iii) On $\mathcal{A}$, the equation can be written as

$$
d u / d t=F(u),
$$

with $F: \mathcal{A} \rightarrow \mathcal{H}$ continuous.

(Note that (ii) does not assume backwards existence: rather, two solutions which exist on $[0, t]$ and agree at time $t$ must have started at the same initial condition.)

Assumptions (i) and (ii) are standard problems in the theory of partial differential equations, whereas (iii) is a new condition that will need to be checked (as do (i) and (ii)) on a case-by-case basis. In the next section an abstract example is treated which shows that the required continuity property for $F$ can be obtained provided the solutions on the attractor are sufficiently smooth.

\subsection{A general parabolic equation}

As an illustration, consider a general parabolic problem, written as an ordinary differential equation on a Hilbert space $H$ (norm $|\cdot|$ and inner product 
$(\cdot, \cdot))$

$$
d u / d t=-A u+g(u)
$$

where $A$ is a positive, unbounded, self-adjoint, linear operator with a compact inverse, and $g(u)$ contains the nonlinear terms. These properties of $A$ guarantee (see Renardy and Rogers (1992), for example) that $A$ has a complete set of orthonormal eigenfunctions $w_{j}$ with eigenvalues $\lambda_{j}$, which can be ordered to be increasing, so that

$$
A w_{j}=\lambda_{j} w_{j} \quad \lambda_{j}>0 \quad \lambda_{j+1} \geq \lambda_{j}
$$

Since $A u$ can be given by the expansion

$$
A u=\sum_{j=1}^{\infty} \lambda_{j}\left(u, w_{j}\right) w_{j}
$$

one can define fractional powers of $A, A^{\alpha}$, in a natural way by setting

$$
A^{\alpha} u=\sum_{j=1}^{\infty} \lambda_{j}^{\alpha}\left(u, w_{j}\right) w_{j}
$$

The domain of $A^{\alpha}$ in $H$, denoted by $D\left(A^{\alpha}\right)$, is all those $u \in H$ for which $\left|A^{\alpha} u\right|$ is finite,

$$
D\left(A^{\alpha}\right)=\left\{u \in H:\left|A^{\alpha} u\right|<\infty\right\} .
$$

Since (5.5) gives an expression for $\left|A^{\alpha} u\right|$,

$$
\left|A^{\alpha} u\right|^{2}=\sum_{j=1}^{\infty} \lambda_{j}^{2 \alpha}\left|\left(u, w_{j}\right)\right|^{2},
$$

an equivalent definition of $D\left(A^{\alpha}\right)$ is

$$
D\left(A^{\alpha}\right)=\left\{u: u=\sum_{j=1}^{\infty} c_{j} w_{j}, \sum_{j=1}^{\infty} \lambda_{j}^{2 \alpha}\left|c_{j}\right|^{2}<\infty\right\} .
$$

Note also that (5.5) implies the bounds

$$
\left|A^{\alpha} u\right| \leq \lambda_{1}^{\alpha-\beta}\left|A^{\beta} u\right| \quad \alpha<\beta .
$$


The fractional powers of $A$ give a convenient way to characterise the continuity of the nonlinear term $g$. Suppose in what follows that $g$ is locally Lipschitz from some space $D\left(A^{\alpha}\right)$ into $H$, so that

$$
|g(u)-g(v)| \leq C(k)\left|A^{\alpha}(u-v)\right| \quad \text { for } \quad\left|A^{\alpha} u\right|,\left|A^{\alpha} v\right| \leq k,
$$

with $C(k)$ an increasing function of $k$.

Assuming now that properties (i) and (ii) hold for (5.4) with $\mathcal{H}=H$, it remains to check (iii), the continuity property of

$$
F(u)=-A u+g(u)
$$

on $\mathcal{A}$. As a function from $H$ into $H, F$ is certainly not continuous, since $A$ is unbounded on $H$.

To obtain continuity on $\mathcal{A}$, it is sufficient to have bounds on all points in $\mathcal{A}$ in a space of sufficiently smooth functions. For example, suppose that $\mathcal{A}$ is a bounded set in $D\left(A^{3 / 2}\right)$, so that

$$
\left|A^{3 / 2} u\right| \leq R \quad u \in \mathcal{A}
$$

A uniform bound on $\mathcal{A}$ in $D\left(A^{\alpha}\right)$ for all $0 \leq \alpha \leq \frac{3}{2}$ now follows from (5.6). (Note that, in what follows, a bound in any space $D\left(A^{\beta}\right), \beta>1$, would be sufficient; the particular exponent $3 / 2$ is chosen to simplify the presentation.)

Setting $x=u-v$, with $x=\sum_{j=1}^{\infty} c_{j} w_{j}$, use Hölder's inequality

$$
\sum_{i=1}^{\infty} a_{i} b_{i} \leq\left(\sum_{i=1}^{\infty} a_{i}^{p}\right)^{1 / p}\left(\sum_{i=1}^{\infty} b_{i}^{q}\right)^{1 / q} \quad p^{-1}+q^{-1}=1
$$

with $p=3$ and $q=3 / 2$, on the expression for $|A x|$,

$$
|A x|^{2}=\sum_{j=1}^{\infty} \lambda_{j}^{2}\left|c_{j}\right|^{2}
$$


to yield

$$
\begin{aligned}
|A x|^{2} & \leq\left(\sum_{j=1}^{\infty}\left|c_{j}\right|^{2}\right)^{1 / 3}\left(\sum_{j=1}^{\infty} \lambda_{j}^{3}\left|c_{j}\right|^{2}\right)^{2 / 3} \\
& =|x|^{2 / 3}\left|A^{3 / 2} x\right|^{4 / 3}
\end{aligned}
$$

Then, (5.8) gives

$$
|A u-A v| \leq(2 R)^{2 / 3}|u-v|^{1 / 3} \quad u, v \in \mathcal{A}
$$

and $A$ is Hölder continuous on $\mathcal{A}$. The Lipschitz continuity of $g$ in (5.7) along with (5.6) now shows that $F$ is continuous on $\mathcal{A}$ as required.

(The solutions of (5.4) considered above are weak solutions, along the lines of (5.3). In his monograph, Henry (1981) shows the existence of "strong" solutions of (5.4), which corresponds to a semigroup on the space $D\left(A^{\alpha}\right)$; so in this case one must take $\mathcal{H}=D\left(A^{\alpha}\right)$. To check (ii) and (iii) now requires additional assumptions. $\alpha \leq \frac{1}{2}$ suffices to give backwards uniqueness (using the argument in Temam (1988, Chapter III section 6)); the attractor needs to be bounded in $D\left(A^{\beta}\right)$ for some $\beta>1+\alpha$ to obtain continuity of $A: \mathcal{A} \rightarrow D\left(A^{\alpha}\right)$; continuity of $g: \mathcal{A} \rightarrow D\left(A^{\alpha}\right)$ then requires continuity of $g$ from $D\left(A^{1+\alpha}\right)$ into $D\left(A^{\alpha}\right)$. Full details will depend on the particular equation.)

\section{The Navier-Stokes equations and turbulence}

The Navier-Stokes equations are the standard model used to govern the flow of a Newtonian fluid. For an incompressible fluid with velocity $u(x, t)$ and pressure $p(x, t)$, in a smoothly bounded open domain $\Omega \subset \mathbb{R}^{n}$ (with $n=2$ or 3 ) under a time-independent body forcing $f_{b}(x)$, the Navier-Stokes equations are:

$$
\frac{\partial u}{\partial t}-\nu \Delta u+u \cdot \nabla u+\nabla p=f_{b} \quad \nabla \cdot u=0
$$

$\nu$ here is the kinematic viscosity. To complete the model, one must also impose the Dirichlet ("no slip") boundary condition $u_{\left.\right|_{\partial \Omega}}=0$. 
To formulate this as an infinite-dimensional dynamical system, one has to identify the correct phase space. For full details of the following mathematical manipulations, see the books by Constantin and Foias (1988) or Temam (1979).

Denote by $\mathbb{L}^{2}(\Omega)=L^{2}(\Omega)^{n}$, the space of vector-valued functions whose $n$ components are in $L^{2}(\Omega)$, with norm

$$
|u|_{\mathbb{L}^{2}}^{2}=\sum_{j=1}^{n} \int_{\Omega}\left|u_{j}\right|^{2} d x
$$

and by $C_{c}^{\infty}(\Omega)$ the space of all infinitely differentiable, compactly supported, $\mathbb{R}^{n}$ valued functions on $\Omega$.

By choosing the phase space $H$ to incorporate the incompressibility condition $(\nabla \cdot u=0)$

$$
H=\text { closure in } \mathbb{L}^{2}(\Omega) \text { of }\left\{u \in C_{c}^{\infty}(\Omega): \nabla \cdot u=0\right\},
$$

the equation can be re-written in functional form, as an ordinary differential equation on $H$,

$$
d u / d t+\nu A u+B(u, u)=f
$$

Here, $A u=-\Pi \Delta u$, where $\Pi$ is the orthogonal projector from $\mathbb{L}^{2}(\Omega)$ onto $H$, $B(u, v)=\Pi(u \cdot \nabla v)$, and $f=\Pi f_{b}$. The operator $A$ is a self-adjoint, positive, linear operator with compact inverse. The norm on $H$, which is just the $\mathbb{L}^{2}$ norm, will be denoted by $|\cdot|$. It is convenient to denote by $V$ the domain of $A^{1 / 2}$, and the norm in $V$ by $\|\cdot\|$,

$$
V=D\left(A^{1 / 2}\right) \quad \text { and } \quad\|u\|=\left|A^{1 / 2} u\right|
$$

This norm is also given by the expression

$$
\|u\|^{2}=\sum_{i, j=1}^{n}\left|\partial_{i} u_{j}\right|^{2},
$$


where $\partial_{i}$ denotes the partial derivative $\partial / \partial x_{i}$.

The properties of the bilinear form $B(u, v)$ are different for $n=2$ and $n=3$, and the two cases need to be treated separately.

\subsection{The Navier-Stokes equations in two dimensions}

For $n=2, B(u, v)$ satisfies the inequalities

$$
|B(u, v)| \leq c \begin{cases}|u|^{1 / 2}\|u\|^{1 / 2}\|v\|^{1 / 2}|A v|^{1 / 2} & u \in V, v \in D(A) \\ |u|^{1 / 2}|A u|^{1 / 2}\|v\| & u \in D(A), v \in V .\end{cases}
$$

These inequalities are sufficiently strong to prove the existence and uniqueness of solutions of (6.1) for initial conditions in $H$. This semiflow possesses a compact global attractor $\mathcal{A}$ as in (i), and also satisfies backwards uniqueness property (ii); details are given in Temam (1988).

To show continuity of

$$
F(u)=-\nu A u+B(u, u)+f,
$$

some regularity of the attractor will be needed, as in the previous section. A result of Guillopé (1982) guarantees that the regularity of $\mathcal{A}$ improves as the smoothness of the forcing term is increased:

Theorem 6.1 (Guillopé). If $f \in D\left(A^{k / 2}\right)$ then the attractor is bounded in $D\left(A^{(k+1) / 2}\right)$.

Assume therefore that $f \in D(A)$, so that the attractor is bounded in $D\left(A^{3 / 2}\right)$ as in (5.8),

$$
\left|A^{3 / 2} u\right| \leq R \quad u \in \mathcal{A}
$$

Then $\nu A$ is continuous from $\mathcal{A}$ into $H$ as argued above (leading to (5.9)), and it only remains to check the continuity of

$$
g(u)=-B(u, u)+f .
$$


First, setting $\mu=\lambda_{1}^{-1 / 2}$ (for ease of notation), note that (6.3) also gives (by (5.6)) the bounds

$$
|A u| \leq \mu R, \quad\|u\| \leq \mu^{2} R, \quad|u| \leq \mu^{3} R, \quad \text { for all } u \in \mathcal{A} .
$$

On $\mathcal{A}, B(u, u)$ is Lipschitz from $V$ into $H$ : writing $w=u-v$,

$$
g(v)-g(u)=B(u, u)-B(v, v)=B(w, u)+B(v, w)
$$

It follows from the inequalities for $B(6.2)$ and the bounds in (6.4) that

$$
\begin{aligned}
|B(u, u)-B(v, v)| & \leq|B(w, u)|+|B(v, w)| \\
& \leq c\left[|w|^{1 / 2}\|w\|^{1 / 2}\|u\|^{1 / 2}|A u|^{1 / 2}+|v|^{1 / 2}|A v|^{1 / 2}\|w\|\right] \\
& \leq 2 c \mu^{2} R\|w\| .
\end{aligned}
$$

Thus, with $C_{1}=2 c \mu^{2} R$,

$$
|B(u, u)-B(v, v)| \leq C_{1}\|u-v\| \quad u, v \in \mathcal{A}
$$

Since $\|w\| \leq \mu|A w|$, the continuity of $A$ on $\mathcal{A}$, (5.9), ensures the required continuity of $F$.

So, provided that $f \in D(A)$, all the assumptions needed in theorem 2.1 are met, and the asymptotic behaviour of the 2D Navier-Stokes equations can be approximated arbitrarily closely by the solutions of a three-dimension system of ordinary differential equations.

\subsection{The Navier-Stokes equations in three dimensions}

The inequalities for $B(u, v)$ in three dimensions,

$$
|B(u, v)| \leq c \begin{cases}|u|^{1 / 4}|A u|^{3 / 4}\|v\| & u \in D(A), v \in V \\ \|u\|\|v\|^{1 / 2}|A v|^{1 / 2} & u \in V, v \in D(A)\end{cases}
$$


have not yet proved sufficient to show existence and uniqueness of solutions in this more important physical case. Whether solutions with smooth initial conditions remain smooth for all time, or whether singularities develop in the flow, is one of the major unsolved problems in the theory of partial differential equations (see Doering and Gibbon (1995) for a nice discussion of the difficulties involved). However, it is possible to derive results by taking the existence and uniqueness of solutions (in some appropriate sense) as the main assumption.

Indeed, a beautiful result due to Constantin, Foias, and Temam (1985) guarantees the existence of a global attractor in $V$ provided that the equations define a semigroup on $V$.

Theorem 6.2 (Constantin, Foias, and Temam). Take $f \in V$. Suppose that the 3D Navier-Stokes equations (6.1) define a semigroup on $V$, so that if $u_{0} \in V$ there exists a unique solution $u(t)$ satisfying $u \in C([0, T] ; V)$ for all $T>0$. Then there is a global attractor $\mathcal{A}$ for the semigroup on $V$.

(Strictly, the existence of a global attractor follows from the result of Foias et al. (1985) (a bounded absorbing set in $V$ ) and some further analysis due to Heywood and Rannacher (1982) that guarantees that the absorbing set is compact in $V$, as required in the infinite-dimensional case.)

As the semigroup is defined on $V$, the phase space $\mathcal{H}$ is $V$, rather than $H$ as before. Given that solutions have the backwards uniqueness property (ii) - which follows from the analysis in Temam (1988) - what remains to be checked is the continuity of

$$
F(u)=-\nu A u+B(u, u)+f
$$

from $\mathcal{A}$ into $V$. 
Since $\mathcal{H}=V, f$ will need to be more regular than in the $2 \mathrm{D}$ case. To obtain regularity of solutions on the attractor, one can again use the result of theorem 6.1, which still holds for $n=3$ provided the assumptions of theorem 6.2 remain. If $f \in D\left(A^{3 / 2}\right)$, theorem 6.1 ensures that the global attractor is bounded in $D\left(A^{2}\right)$,

$$
\left|A^{2} u\right| \leq R \quad u \in \mathcal{A}
$$

this allows the previous argument to be used to show the continuity of $A: \mathcal{A} \rightarrow V$,

$$
\|A u-A v\| \leq(2 R)^{2 / 3}\|u-v\|^{1 / 3} \quad u, v \in \mathcal{A} .
$$

What remains is to check the continuity properties for the nonlinear part of $F(u)$, $B(u, u)$. To derive bounds for $\|B(u, v)\|$ from those for $|B(u, v)|$, note that

$$
\partial_{i} B(u, v)=B\left(\partial_{i} u, v\right)+B\left(u, \partial_{i} v\right)
$$

and it then follows from (6.5) that

$$
\|B(u, v)\| \leq k\left\{\begin{array}{r}
\|u\|^{1 / 4}\left|A^{3 / 2} u\right|^{3 / 4}\|v\|+|u|^{1 / 4}|A u|^{3 / 4}|A v| \\
u \in D\left(A^{3 / 2}\right), v \in D(A), \\
|A u|\|v\|^{1 / 2}|A v|^{1 / 2}+\|u\|\left|A^{3 / 2} v\right|^{1 / 2}|A v|^{1 / 2} \\
u \in D(A), v \in D\left(A^{3 / 2}\right) .
\end{array}\right.
$$

A little algebra, exactly following the two-dimensional case, now shows that

$$
\|B(u, u)-B(v, v)\| \leq 4 k \mu^{5 / 2} R|A w|=C_{1}\left\|A^{1 / 2} w\right\|
$$

and the required continuity follows from (6.6).

Application of theorem 2.1 now shows that, provided that the Navier-Stokes equations are indeed a good model for fluid flow (in the sense of theorem 6.2), and that the forcing is sufficiently smooth $\left(f \in D\left(A^{3 / 2}\right)\right)$, fully-developed threedimensional turbulence can be approximated arbitrarily closely by a system with only three degrees of freedom. 


\section{Assorted open problems}

This section covers, briefly, several topics that deserve further attention. For simplicity the discussion will be restricted to the finite-dimensional case, when the original system - "the $F$ system" - is in $\mathbb{R}^{n}$, and reduced to a three-dimensional "f system".

\subsection{Properties of pseudo-orbits}

Any numerical scheme to approximate the solutions of the $F$ system will in fact generate some manner of pseudo-orbit (cf. section 2), as does the $f$ system. It is therefore very important to discover exactly how much information can be obtained about a system from its pseudo-orbits. Classical shadowing results work for hyperbolic attractors (Bowen 1975), and there are some recent extensions to long-time shadowing results for non-hyperbolic systems (Coomes et al. 1995), but there is a need for a general theory which covers the relationship between the attractors (and recurrent sets) for a system and its pseudo-orbits.

\subsection{Dynamic behaviour of the three-dimensional system}

One might suspect that the reflection of the dynamics of the $F$ system by those of the $f$ system is entirely dependent on the function $\Phi$; however, corollary 2.2 , which ensures that the image under $\Phi$ of pseudo-orbits of the $f$ system shadow complete orbits of the $F$ system, shows that this in not the case, and that one can

indeed expect that the $f$ system gives good information about the behaviour of the $F$ system.

It would therefore be of interest to study the dynamics of the $f$ system, for example the structure of its attractor. The embedded trajectories (those used to approximate the $F$ system) may in fact be only transients in the $f$ system as 
constructed in this article; if they are, it may be possible to modify the construction to ensure that they lie on the attractor.

It is noteworthy that a three-dimensional dissipative system can have only one positive Lyapunov exponent. Since the result of this paper implies that this should be sufficient to capture the dynamics of any higher-dimensional system, it should be possible to observe phenomena which may have appeared to be intrinsic to "hyper chaos" (two positive Lyapunov exponents) in chaotic three-dimensional systems.

A similar comment can be made about the dimension of the global attractor of partial differential equations, which has become a standard measure of their complexity (e.g. Temam 1988). Although this is reflected in constructions which attempt to reproduce the dynamics on $\mathcal{A}$ exactly (Eden et al. 1994, Robinson 1997), the approximation result here casts some doubt on the idea that the higher the dimension the more intrinsically complex the system.

On a more positive note, the theory of knots (Holmes 1988) can be applied to three-dimensional flows, but not to flows in higher dimensions. It might be possible to use the $f$ system in some way to discuss the "knotting" of solutions in the $F$ system.

\subsection{Computing attracting sets}

Often numerical experiments with many degrees of freedom produce pictures of the attractor which bear some resemblance to attractors from familiar threedimensional systems, like the Lorenz attractor (Lorenz 1963, Sparrow 1982) or the Rössler attractor (Rössler 1976). In the light of the result of this paper this is unsurprising. 
Indeed, suppose that $\mathcal{A}$ is the attractor of some high-dimensional system (in $\mathbb{R}^{n}$ ), with $|x| \leq R$ for all $x \in \mathcal{A}$. Assuming that there is a dense orbit $\tau \subset \mathcal{A}$, a computer-generated image of the attractor is obtained by calculating a numerical version of this trajectory, $\tau_{n}$, and then projecting it onto a twodimensional subspace, via a projection $\Pi_{2}$. The "picture of the attractor" so obtained is therefore $\Pi_{2} \tau_{n}$; assume that this picture lies within $\epsilon$ of the "true picture" $\Pi_{2} \tau$.

An argument based on the proof of theorem 2.1 shows that one can obtain essentially the same picture (a two-dimensional representation within $\epsilon$ of $\Pi_{2} \tau$ ) starting with a three-dimensional system.

Extend the projection $\Pi_{2}$ by adding some arbitrary third component, to give a projection $\Pi_{3}: \mathbb{R}^{n} \rightarrow \mathbb{R}^{3}$. As remarked in section 3 , the embedding result (theorem 3.1) holds for a dense set of projections. It follows that there is a projection $P_{3}=P_{1}+P_{2}$ (where the rank of $P_{i}$ is $i$ ), injective on $\tau$, such that

$$
\left\|P_{3}-\Pi_{3}\right\| \leq \epsilon / 3 R
$$

and also

$$
\left\|P_{2}-\Pi_{2}\right\| \leq \epsilon / 3 R
$$

(7.1) ensures that $\Pi_{3} \tau$ and $P_{3} \tau$ differ by at most $\epsilon / 3$. The construction from the proof of theorem 2.1 can be used to produce a Lipschitz vector field with a solution $\tau_{l}$ that approximates $P_{3} \tau$ to within $\epsilon / 3$, and so $\Pi_{3} \tau$ within $2 \epsilon / 3$. Now (7.2) shows that $P_{2} \tau_{l}$ approximates $\Pi_{2} \tau$ to within $\epsilon$, obtaining "the same" picture of the attractor as before from a solution of a three-dimensional system.

\subsection{Bifurcations and continuity with respect to parameters}

Since the study of bifurcations is fundamental to the theory of dynamical 
systems, it is natural to ask how the $f$ system is affected by a change of parameters in the $F$ system. As an indication that it is not unreasonable to expect to be able to construct a continuously varying three-dimensional $f$ system, it is shown below that one can construct a continuously varying five-dimensional system of equations whose pseudo-orbits follow a continually varying one-parameter family $F_{\mu}$.

First, restrict attention to some interval $\mathcal{I}$ of $\mu$ values, and assume that $F_{\mu}$ varies continuously with $\mu$, so that the corresponding solutions vary in a Lipschitz way,

$$
\left|\varphi_{\mu_{1}}(t) u_{0}-\varphi_{\mu_{2}}(t) u_{0}\right| \leq K(T)\left|\mu_{1}-\mu_{2}\right| \quad t \in[0, T]
$$

for each $T>0$.

Rather than approximate orbits on the attractors $\mathcal{A}_{\mu}$, which will generally vary with $\mu$, assume that there is a compact set $B$ which is absorbing for all $\mu \in \mathcal{I}$, and that the separation of trajectories within $B$ (as in (1.3)) can be taken to be uniform over this range. In this case, there is a fixed (finite) set of $\left\{u_{k}\right\} \in B$ such that, for any $u_{0} \in B$, there is a $u_{k}$ with

$$
\left|\varphi_{\mu}(t) u_{0}-\varphi_{\mu}(t) u_{k}\right| \leq \epsilon / 2 \quad t \in[0, T]
$$

and this is true for all $\mu \in I$.

As before, define the orbit segment $\tau_{k}^{\mu}$ through $u_{k}$ due to the flow $\varphi_{\mu}$,

$$
\tau_{k}^{\mu}=\bigcup_{0 \leq t \leq T} \varphi_{\mu}(t) u_{k}
$$

and consider the collection of these, $\tau_{k}^{M}$, over $\mu \in \mathcal{I}$ :

$$
\tau_{k}^{M}=\bigcup_{\mu \in \mathcal{I}} \tau_{k}^{\mu}
$$


Since $\tau_{k}^{M}$ is the Lipschitz image of $[0, T] \times \mathcal{I}$, it has fractal dimension at most 2 . Defining $\mathcal{T}^{\mu}$ as before (the collection of all the $\tau_{k}^{\mu}$ ),

$$
\mathcal{T}^{\mu}=\bigcup_{k} \tau_{k}^{\mu}
$$

note that the collection of these over $\mu$,

$$
\mathcal{T}^{M}=\bigcup_{\mu \in \mathcal{I}} \mathcal{T}^{\mu}
$$

is also given by

$$
\mathcal{T}^{M}=\bigcup_{k} \tau_{k}^{M}
$$

and so has $d_{F}\left(\mathcal{T}^{M}\right) \leq 2$.

Theorem 2.1 now shows that there is a projection $P: \mathbb{R}^{n} \rightarrow \mathbb{R}^{5}$ injective on $\mathcal{T}^{M}$, and hence injective on each $\mathcal{T}^{\mu}$. The inverse of $P$ is well-defined on $P \mathcal{T}^{M}$, and Hölder continuous there. Now define

$$
g_{\mu}(x)=P F_{\mu}\left(P^{-1} x\right) \quad \text { for } \quad x \in P \mathcal{T}^{M}
$$

since $P$ is fixed, $g_{\mu}$ varies continuously with $\mu$ over its domain of definition $\left(P \mathcal{T}^{M}\right)$. Note that the extension $E_{X}(f)$ of $f$ to the whole of $\mathbb{R}^{m}$ provided by theorem 3.2 is a continuous function of $f$. Thus one can extend the functions $g_{\mu}(x)$ to a continuously varying family $G_{\mu}$ defined on the whole of $\mathbb{R}^{5}$.

The equations $d x / d t=G_{\mu}(x)$ are not necessarily Lipschitz, so uniqueness is lost (it is precisely this which entailed the additional construction in the proof of theorem 2.1). However, they are a continuous family in a phase space of muchreduced dimension, pseudo-trajectories of which, under the constant (with respect to $\mu \in \mathcal{I}$ ) mapping $\Phi=P^{-1}$, give the changing dynamics of the original $F_{\mu}$ systems. 
To obtain a continuously varying three-dimensional Lipschitz system, one would need to investigate further the dependence of the injective projections of theorem 3.1 on the set $X$, the continuity of the extension $E_{X}(f)$ with respect to the set $X$, and the relationship of the Lipschitz $f$ constructed in the proof of theorem 2.1 to the non-Lipschitz $g$ used above.

\section{Conclusion}

Theorem 2.1 is, essentially, applicable to any dissipative ordinary differential equation, and to many partial differential equations. The result shows that any trajectory of such systems can be shadowed to within an $\epsilon$ error by the continuous image of a $(T, 2 \epsilon)$ pseudo-orbit of a three-dimensional system.

That the behaviour of so many systems can be investigated by considering a three-dimensional set of equations provides a justification for all the many investigations into such easily-visualised models, and a spur to their further study.

\section{Acknowledgments}

Many thanks to Trinity College, Cambridge, for their financial support and continual generosity over the past four years. Thanks to Ciprian Foias, José Langa, and Colin Sparrow, for both their interest and their helpful suggestions. 


\section{References}

Agmon S, Douglis A and Nirenberg L 1964 Estimates near the boundary for solutions of elliptic partial differential equations satisfying general boundary conditions II Commun. Pure Appl. Math. 17 35-92

Billotti J E and LaSalle J P 1971 Dissipative periodic processes Bull. Amer. Math. Soc. 77 1082-1088

Constantin P, Foias C and Temam R 1985 Attractors representing turbulent flows Mem. Amer. Math. Soc. 53

Constantin P and Foias C 1988 Navier-Stokes equations (Chicago: University of Chicago Press)

Coomes B A, Koçak H and Palmer K J 1995 Rigorous computational shadowing of orbits of ordinary differential equations Numer. Math. 69 401-421

Doering C R and Gibbon J D 1995 Applied Analysis of the Navier-Stokes Equations (Cambridge: Cambridge University Press)

Eden A, Foias C, Nicolaenko B and Temam R 1994 Exponential attractors for dissipative evolution equations (Chichester: Wiley)

Falconer K 1990 Fractal Geometry (Chichester: Wiley)

Foias C and Olson E J 1996 Finite fractal dimension and Hölder-Lipschitz parametrization Indiana Univ. Math. J. 45 603-616

Glendinning P 1988 Global bifurcations in flows New Directions in Dynamical Systems ed Bedford T and Swift J (Cambridge: Cambridge University Press) pp 120-149 
Guillopé C 1982 Comportement á l'infini des solutions des équations de NavierStokes et propriété des ensembles fonctionnels invariantes (ou attracteurs) Ann. Inst. Fourier (Grenoble) 32(3) 1-37

Hale J K 1988 Asymptotic Behavior of Dissipative Systems (Mathematical Surveys and Monographs 25) (Providence, RI: American Mathematical Society)

Hartman P 1964 Ordinary Differential Equations (New York: Wiley)

Henry D 1981 Geometric Theory of Semilinear Parabolic Equations (Lecture Notes in Mathematics 840) (New York: Springer-Verlag)

Heywood J G and Rannacher R (1982) Finite approximation of the nonstationary Navier-Stokes problem, Part I: Regularity of solutions and second-order error estimates for spatial discretization SIAM J. Num. Anal. 19 275-311

Holmes P 1988 Knots and orbit genealogies in nonlinear oscillators New Directions in Dynamical Systems ed Bedford T and Swift J (Cambridge: Cambridge University Press) pp 150-191

Katok A and Hasselblatt B 1995 Introduction to the Modern Theory of Dynamical Systems (Cambridge: Cambridge University Press)

Langa J A and Robinson J C 1996 Determining asymptotic behaviour from the dynamics on attracting sets (submitted)

Lorenz E 1963 Deterministic nonperiodic flow J. Atmospheric Sci. 20 130-141

Mañé R 1981 On the dimension of the compact invariant sets of certain nonlinear maps, Springer Lecture Notes in Mathematics 898 230-242

Mielke A 1997 The complex Ginzburg-Landau equation on large and unbounded 
domains: sharper bounds and attractors Nonlinearity 10 199-222

Nicolaenko B, Scheurer B and Temam R 1985 Some global properties of the Kuramoto Sivashinksy equation: Nonlinear stability and attractors Physica 16D $155-183$

Renardy M and Rogers R C 1992 An Introduction to Partial Differential Equations (Texts in Applied Mathematics 13) (New York: Springer)

Robinson J C 1997 Global attractors: topology and finite-dimensional dynamics J. Dynam. Diff. Eqns (to appear)

Rössler O E 1976 An equation for continuous chaos Physics Letters A 57 397-398

Sparrow C 1982 The Lorenz Equations: Bifurcations, Chaos, and Strange Attractors (New York: Springer)

Stein E M 1970 Singular integrals and differentiability properties of functions (Princeton, NJ: Princeton University Press)

Stuart A M and Humphries A R 1996 Dynamical Systems and Numerical Analysis (Cambridge: Cambridge University Press)

Temam R 1979 Navier-Stokes Equations, Theory and Numerical Analysis (Amsterdam: North Holland)

Temam R 1988 Infinite Dimensional dynamical systems in Mechanics and Physics (Applied Mathematics Sciences 68) (New York: Springer) 